\title{
Distal Interphalangeal Joint 4
}

National Cancer Institute

\section{Source}

National Cancer Institute. Distal Interphalangeal Joint 4. NCI Thesaurus. Code C102293.

A ginglymoid (hinge) synovial joint within the fourth digit of the hand or foot connecting the middle and distal phalanges. 